\title{
THE OPTIMAL IMPLEMENTATION POLICY FOR INSPECTING PAVEMENT WITH DETERIORATION UNCERTAINTY
}

\author{
Kiyoshi KOBAYASHI ${ }^{1}$, Masayuki EGUCHI ${ }^{2}$, Akira OI ${ }^{3}$, Kazuya AOKI ${ }^{4}$ \\ and Kiyoyuki KAITO ${ }^{5}$ \\ ${ }^{1}$ Fellow, Graduate School of Management, Kyoto Univ. (Yoshida-honmachi, Sakyo-ku, Kyoto, 606-8501) \\ E-mail: kobayashi.kiyoshi.6n@kyoto-u.ac.jp \\ ${ }^{2}$ Member of JSCE, East Nippon Expressway Company Limited, Technology \& Environment Department \\ (3-3-2 Kasumigaseki, Chiyoda-ku, Tokyo, 100-8979) \\ E-mail:m.eguchi.ac@e-nexco.co.jp \\ ${ }^{3}$ Member of JSCE, Central Nippon Expressway Company Limited, Hachioji Branch \\ (2858 Saijo, Showa, Nakakoma, Yamanashi, 409-3866) \\ E-mail:a.oi.aa@c-nexco.co.jp \\ ${ }^{4}$ Member of JSCE, PASCO Corporation, Infrastructure Management Division \\ (2-6-17 Aobadai, Aoba-ku, Yokohama, 227-0062) \\ E-mail: kiakzo6013@pasco.co.jp \\ ${ }^{5}$ Member of JSCE, Dept. of Civil Eng., Osaka Univ. (2-1 Yamada-oka, Suita, Osaka, 565-0871) \\ E-mail: kaito@ga.eng.osaka-u.ac.jp
}

\begin{abstract}
This study proposes a methodology to find the optimal inspection policy for road pavement with uncertain deterioration processes. Since the deterioration progresses are characterized by a lot of uncertainty, the pavement conditions cannot be deterministically evaluated unless the inspection or repair activities are carried out. The road administrators have to decide whether repair should be implemented based upon the results of inspection. In this paper, the value of the inspection is evaluated by using the real option theory, and the optimal inspection and repair model is formulated to investigate the optimal inspection frequencies and repair policy, which may minimize the expected life cycle costs. Finally, the validity of the methodology presented in the paper is investigated by a case study dealing with the expressway in the real world.
\end{abstract}

Key Words : pavement management, inspection policy, real option, state-dependent rules

\section{INTRODUCTION}

The deterioration process of road pavement includes significant uncertainty, and it is difficult to accurately predict the state of future deterioration of pavement. As statistical deterioration prediction models considering such uncertainty of the deterioration process of pavement, Markov deterioration models, etc. have been proposed $^{1-7)}$. By using a statistical deterioration prediction model, it became possible to predict the deterioration process according to actual conditions, based on the results of actual surveys on road properties. This improved the reliability of the lifecycle cost estimation for pavement structures, pavement materials, and repair methods.

The analysis of repair policies based on lifecycle costs provides the information on repair poli- cies at the macro level summarizing individual road spans, such as budgetary plans and repair standards at the network level. However, when micro repair policies are designed for individual road spans, it is necessary to discuss repair methods and timings based on the soundness level of individual roads. A lot of external factors influence the deterioration process of pavement, and so it is extremely difficult to predict the deterioration process of pavement for individual road spans. To determine whether to maintain or repair each road pavement, it is essential to monitor its soundness level through road condition surveys, etc.

To obtain information on the soundness level of pavement, the cost of road condition surveys and social costs for traffic control, etc., must be considered. To curb the monitoring cost, it is desirable to reduce the frequency of road condition 
surveys. As time passes, the uncertainty in the soundness level of pavement augments. In addition, there is a possibility that the pavement will deteriorate further and the cost for repairing it will increase. Accordingly, we require a methodology for determining the inspection cycle and pavement repair strategies for minimizing lifecycle costs, including repair costs, social expenses, and monitoring expenditures, under the risk control level for pavement services specified by road administrators.

Accordingly, the authors express the deterioration process of pavement with a Markov deterioration model and formulate a Markov decision model (hereinafter, optimal inspection/repair model) for determining both inspection cycle and a repair policy for minimizing the lifecycle cost under a specified risk control level. Chapter 2 describes the basic idea of this study. Chapter $\mathbf{3}$ formulates the optimal inspection/repair model. Chapter 4 formulates an extended model considering additional surveys. Chapter 5 discusses the economic benefits of road condition surveys. Chapter 6 shows a case of empirical analysis targeted at actual expressways.

\section{BASIC IDEA OF THIS STUDY}

\section{(1) Review of previous studies}

We can enumerate two kinds of repair strategies for maintaining and repairing civil infrastructures: (1) the time-dependent rule and (2) the condition-dependent rule. The time-dependent rule is the method of maintaining and renewing civil infrastructures at certain intervals. For systems composed of a lot of small-scale equipment, such as lighting systems and other road facility systems, and civil infrastructures whose inspection cost ratio is high, including buried objects, such as pipes, it is desirable to adopt the timedependent rule for renewing civil infrastructures and equipment regularly regardless of their deterioration states. On the other hand, if the deterioration process includes significant uncertainty, it is desirable to adopt the condition-dependent rule for determining repair policies according to the deterioration state of civil infrastructures. In this study, the authors focus on the uncertainty of the deterioration process of road pavement, and propose the inspection/repair policy using the condition-dependent rule.

There has been a large number of researches into the optimal repair strategies of a system in which damage or failure occurs in accordance with the stationary stochastic process ${ }^{8)}$. Especially, with the Markov decision model ${ }^{9)}$, in which soundness level is expressed by a discrete state variable, the deterioration process can be easily described, and so many practical models ${ }^{10)}-18$ ) have been proposed in the civil engineering field. The soundness level of most civil infrastructures can be obtained partially through inspection only. Researchers have proposed some methods for analyzing the facilities whose deterioration process cannot be observed directly by using the Markov decision process. In these methods, inspection timing is predetermined, and the investment in repair is determined based on the data obtained through periodic inspections. Especially, the costs for inspecting buried civil infrastructure cannot be ignored. In this case, it is important to determine inspection timing. Thus, the optimal inspection/repair models for determining inspection cycle and repair policies have been proposed ${ }^{15)-18)}$. In this study, the authors formulated the optimal inspection/repair model as a basic model for the management of road pavement. Furthermore, additional inspection was conducted for the deteriorated parts detected through regular inspection, and the authors formulated the optimal inspection/repair model considering additional inspection/repair policies as an extended model. With the opportunity to carry out additional inspection and repair for limited parts, it is possible to reduce the costs for inspection and repair. Such an extended model can be formulated as the Markov decision model including the sub-Markov decision model for designing additional inspection/repair policies.

\section{(2) Uncertainty of the deterioration pro- cess and the purpose of inspection}

The deterioration of road pavement includes significant uncertainty, and so it is impossible to predict the deterioration process accurately. Here, it is assumed that the road condition inspection is conducted at the initial timing $t=0$ and the soundness level of the inspected pavement is expressed by $h(0)$. As time passes from the initial timing $t=0$, the pavement deteriorates in accordance with the process shown in Fig.1. Fig.1 shows some sample paths of pavement deterioration. Since the deterioration process of pavement includes uncertainty, the uncertainty of the deterioration state increases with time. It is assumed that the road condition is inspected at a certain timing $t$. By inspecting the road condition, it is possible to obtain the soundness level of pavement at the inspection timing. The soundness level of pavement at $t>0$ is represented by $h(t)$. When time passes from the timing $t$, the pavement de- 
teriorates. Road administrators estimate the distribution of the soundness level of pavement at $t^{\prime}\left(t^{\prime}>t\right)$. The figure shows the probability distribution of soundness level $F^{1}$, which can be estimated by road administrators when the road condition is not inspected at $t$, and the probability distribution $F^{2}$ estimated with the data obtained through the road condition inspection at $t$. By inspecting the road condition at $t$, it is possible to reduce the uncertainty of soundness level at $t^{\prime}$ (variance of the probability distribution $F$ ). Here, it is assumed that road administrators set the risk control goal of curbing the probability that the soundness level of pavement will become lower than the specified risk control level. In the case of Fig.1, at $t^{\prime}$, the probability that soundness level will be lower than a specified risk control level is defined by the area of the shaded region of $F^{1}$ and $F^{2}$. By inspecting the road condition, it is possible to remove the uncertainty of soundness level at the inspection timing. In addition, it is possible to reduce the uncertainty after the inspection timing and decrease the lifecycle cost for the reasons described in the following section.

\section{(3) Inspection/repair rule}

It is assumed that the soundness level of pavement is observed by inspecting the road condition regularly and judgment is made whether to repair road pavement based on the results of the road condition inspection. That soundness level turned out to be $a$ through the road condition inspection at $t$ in Fig.1. Road administrators consider two actions: (1) to put off road repair and judge whether to repair the road based on the results of the next road condition inspection (action $e_{1}$ ), or (2) to repair the road immediately (action $e_{2}$ ). If a road administrator chooses action $e_{2}$, soundness level will return to the healthy level (initial state). If action $e_{1}$ is adopted, the road will deteriorate during a period from $t$ to the next periodic inspection timing $t^{\prime}$. If repair is not conducted at $t$, it is impossible to predict the soundness level at $t^{\prime}$ accurately. However, it is possible to estimate the probability distribution of soundness level $F^{2}$ at $t^{\prime}$ with the data on soundness level at $t$, which is obtained through periodic inspection. In this study, pavement management is aimed at curbing the probability that soundness level will become lower than a specified risk control level. Needless to say, as the deterioration state at $t$ worsens, the probability that soundness level will become lower than the risk control level at the next regular inspection timing $t^{\prime}$ when repair is put off increases. As represented by point

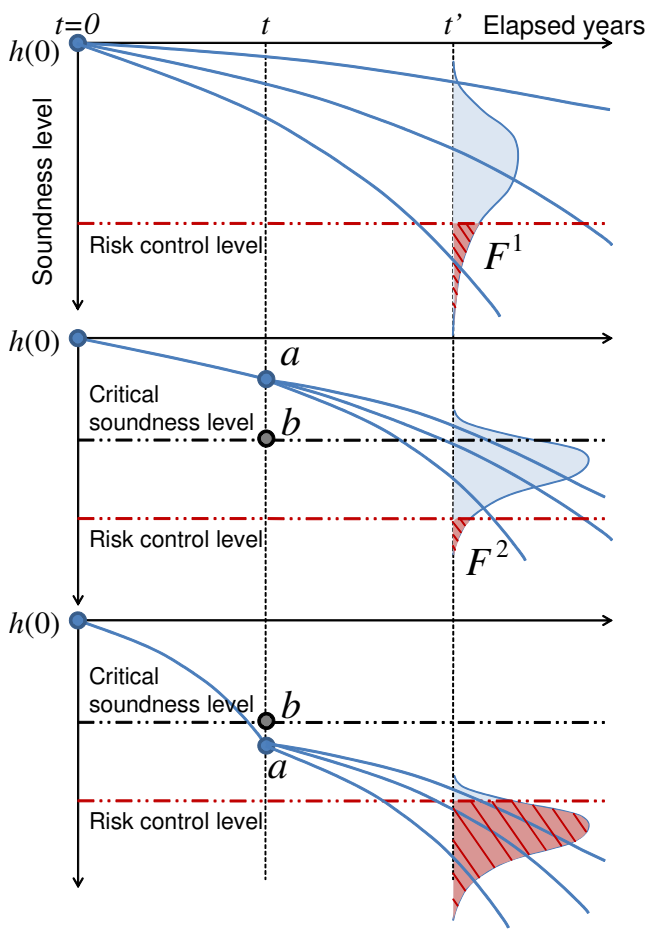

Note: The upper graph shows the case in which the road condition is not inspected at the timing $t$. The middle graph shows the case in which the road condition is inspected at the timing $t$ and the observed soundness level is $a$. The lower graph shows the case in which the deterioration state $a$ at the timing $t$ is lower than the critical soundness level (point $b$ ), and so repair is put off, and then the probability that it will be lower than the risk control level at the timing $t^{\prime}$ increases.

Fig.1 Uncertainty of the deterioration process.

$b$ in Fig.1, there is the critical soundness level below which the management goal is impossible to be achieved at the next regular inspection timing if repair is put off. This critical soundness level is defined as a repair policy for specifying repair standards. Specifically, repair policies can be described as rules, such as put off repair if soundness level is higher than the critical soundness level or conduct repair if soundness level is lower than the critical soundness level at the time of regular inspection.

In the above discussion, it was assumed that regular inspection interval had been specified. If inspection is conducted frequently (inspection cycle is shortened), the probability that the soundness level of pavement will be lower than the risk control level at the next regular inspection timing decreases. Under the assumption that the repair method is unchanged without depending on 
the pavement state, it is desirable that the critical soundness level for judging whether to conduct repair is low so as to decrease the cost for pavement repair. Accordingly, to curb repair expenses, it is optimal to shorten the interval of regular inspection. On the other hand, if inspection interval shortens, inspection expenses augment. Therefore, to decrease the lifecycle cost, it is necessary to determine the interval of regular inspection and a repair policy at the same time to minimize the lifecycle cost, which is composed of inspection and repair expenses. When road administrators consider the minimization of social expenses, it is ideal to discuss the minimization of lifecycle cost, including the user costs. However, at present, precision in the estimation of the user costs is insufficient compared with that for the direct costs for inspection and repair borne by road administrators. In this study, it was assumed that lifecycle costs did not include the user costs. Instead, considering the safety and comfort for users comprehensively, a risk control level for the soundness level of pavement was specified.

\section{(4) Risk-cost management curve}

A lifecycle cost and a risk control level are focused on, as the items of road pavement management. A risk control level is defined by using the probability that the service level of pavement satisfies the control level $\bar{U}$, as formulated in Section 3.(2). Here, a risk control level is fixed at a specified value $\bar{U}$. At this time, each road administrator must determine regular inspection interval and critical soundness level (repair policy) so as to minimize lifecycle cost, under the specified rick control level. The optimal inspection interval and repair policy determined with the above way of thinking are the conditional optimal inspection/repair policy with a specified risk control level. By considering the risk control level as a parameter and obtaining the conditional optimal inspection/repair policy for each risk control level, we can draw the curve representing the trade-off relation between risk control level and lifecycle cost as shown in Fig.2. In this study, such a curve is called the risk-cost management curve. Since the deterioration process of pavement includes uncertainty, if a rigorous risk control level is set as represented by point $\alpha$, frequency inspection becomes necessary, increasing the lifecycle cost. In practice, we have no choice but to tolerate some risks. On the other hand, if the state is at point $\beta$ in the figure, the reduction in inspection cost would decrease the risk control level significantly. If a road administrator specifies a risk control level for the probabil-

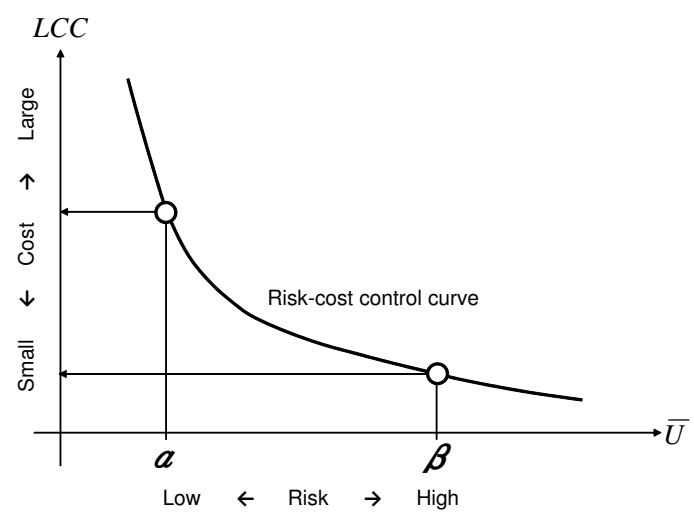

Fig.2 Risk-cost control curve.

ity of attaining the service level, it is possible to obtain the optimal inspection interval and repair policy. Each road administrator needs to consider the convenience and the risk of management failure, etc. comprehensively, when specifying a risk control level. The problem of specifying a risk control level is beyond the scope of this study, but the risk-cost management curve fulfills important roles for road administrators to determine an appropriate risk control level.

\section{BASIC MODEL}

\section{(1) Preconditions for model development}

Assuming the axis of discrete time $t=$ $0,1,2, \cdots$ with the calendar time $s_{0}$ equal to the initial timing, a point on the discrete time axis is called a timing, distinguishing it from calendar time. Unit time span is normalized to one. The soundness level of pavement is expressed by $J$ ratings $i(i=1, \cdots, J)$. As $i$ increases, deterioration progresses. The soundness level of pavement at $t$ is expressed by the state variable $h(t)=i(i=1, \cdots, J ; t=0,1, \cdots) . \quad J$ is the absorbing state. Under the assumption that the deterioration process follows the Markov chain, the transitional probability of soundness level in the unit time span on the discrete time axis is expressed by the Markov transitional probability. The Markov transitional probability is defined by the conditional probability that the soundness level $h(t+1)=j(j \geq i)$ at the timing $t+1$, given that the soundness level observed through the road condition inspection at the timing $t h(t)=i$ :

$$
\operatorname{Prob}[h(t+1)=j \mid h(t)=i]=p_{i j}
$$

By defining such a transitional probability for every pair of soundness levels $(i, j)$, it is possible to define the Markov transitional probability ma- 
trix:

$$
\boldsymbol{p}=\left(\begin{array}{ccc}
p_{11} & \cdots & p_{1 J} \\
\vdots & \ddots & \vdots \\
0 & \cdots & p_{J J}
\end{array}\right)
$$

The Markov transitional probability (1) is the transitional probability of soundness level between the two specified timings $t$ and $t+1$, and it varies according to inspection interval. Since the road keeps deteriorating unless it is repaired, $p_{i j}=0(i>j)$. In addition, because of the definition of transitional probability, $\sum_{j=i}^{J} p_{i j}=1$, specifically, the following conditions must be satisfied:

$$
\left.\begin{array}{l}
p_{i j} \geq 0(i, j=1, \cdots, J) \\
p_{i j}=0(\text { when } i>j) \\
\sum_{j=i}^{J} p_{i j}=1
\end{array}\right\}
$$

State $J$ is the absorbing state in the Markov chain unless repair is not conducted, and $p_{J J}=1$. The Markov transitional probability does not depend on the history of deterioration. The Markov transitional probability model satisfies the characteristic (Markov property) that the probability of the transition from $i-1$ to $i$ during a period from $t$ to $t+1$ depends on only the soundness level at the timing $t$. By using the Markov transitional probability, the deterioration process between the inspection timing $t$ and the next timing $t+r$ can be expressed by the transitional probability matrix

$$
\boldsymbol{p}(r)=\{\boldsymbol{p}\}^{r}
$$

Each element of the transitional probability matrix $\boldsymbol{p}(r)$ in the $r$ duration is expressed by $p_{i j}(r)(i, j=1, \cdots, J)$. For simplicity,

$$
\boldsymbol{p}(0)=\boldsymbol{I}
$$

where $\boldsymbol{I}$ represents $(J \times J)$ unit matrix.

\section{(2) Inspection/repair policy}

It is assumed that the road condition is inspected at $r$ intervals. $r$ is a policy variable, but it is assumed that $r$ is specified. The timing of the $k$-th road condition inspection on the discrete time axis is defined as $t_{k}^{r}=r k(k=0,1, \cdots)$. It is assumed that the $k$-th road condition inspection has revealed that the soundness level of pavement at a certain point is $h\left(t_{k}^{r}\right)=i(i=1, \cdots, J)$. If it is judged that soundness level is $h\left(t_{k}^{r}\right)=J$ at the timing $t_{k}^{r}$, the road is repaired immediately and its soundness level recovers to 1 . On the other hand, the possible actions $e$ when the soundness level at the timing $t_{k}^{r}$ is $1<h\left(t_{k}^{r}\right)<J$ include: (1) to record the results of soundness evaluation without repair work (action $e_{1}$ ) and (2) to conduct repair to recover the soundness level of pavement to 1 (action $e_{2}$ ). The repair policy $\xi$ at the timing of regular inspection is defined by using rule $\eta^{\xi}(i)$ for specifying the soundness level after repair with respect to the soundness level observed in inspection $h\left(t_{k}^{r}\right)=i$. Specifically, the repair policy $\xi$ can be expressed as follows:

$$
\begin{aligned}
& \eta^{\xi}(i)= \begin{cases}i & \left(\text { in the case of action } e_{1}\right) \\
1 & \text { (in the case of action } \left.e_{2}\right)\end{cases} \\
& (i=1, \cdots, J)
\end{aligned}
$$

There exists a threshold soundness level (critical soundness level) $i^{*}(\xi)$, and if the soundness level $i$ reaches or exceeds the critical soundness level $i^{*}(\xi)$, repair is carried out without fail. On the other hand, until it reaches the critical soundness level, repair is not conducted. It is assumed that such a monotonicity condition is satisfied. The monotonicity condition is expressed as follows:

$$
\begin{aligned}
& \eta^{\xi}(i)= \begin{cases}i & i<i^{*}(\xi) \\
1 & i \geq i^{*}(\xi)\end{cases} \\
& (i=1, \cdots, J)
\end{aligned}
$$

A set of repair policies satisfying the monotonicity condition (7) is expressed by $\Xi$. When the repair policy $\xi \in \Xi$ is implemented, repair is conducted immediately according to the soundness level after inspection. The transitional state of soundness level before and after the repair action is defined by the following expression:

$$
\begin{aligned}
& q_{i j}^{\xi}= \begin{cases}1 & \eta^{\xi}(i)=j \\
0 & \text { otherwise }\end{cases} \\
& (i, j=1, \cdots, J)
\end{aligned}
$$

The transitional matrix with $q_{i j}^{\xi}$ equal to the $(i, j)$ element (hereinafter, repair transitional matrix) is expressed by $\boldsymbol{q}^{\xi}$.

In the above discussion, it is assumed that the interval of road condition inspection $r$ has been specified. However, the inspection interval $r$ and the repair policy $\xi$ at the inspection timing are both policy variables. The combination of these two policy variables $(r, \xi) \in \Xi$ is called an inspection/repair policy. Here, $\Xi$ is a set of inspection/repair policies. The deterioration/repair process arising out of the inspection/repair policy $(r, \xi) \in \Xi$ can be defined with the transitional probability $P_{i j}^{\xi}(r)$ :

$$
P_{i j}^{\xi}(r)=\sum_{k=1}^{J} q_{i k}^{\xi} p_{k j}(r)
$$

The transitional probability matrix with $P_{i j}^{\xi}(r)$ equal to the $(i, j)$ element is expressed by $\boldsymbol{P}^{\xi}(r)$. The matrix notation of Equation (9) is as follows:

$$
\boldsymbol{P}^{\xi}(r)=\boldsymbol{q}^{\xi} \boldsymbol{p}(r)
$$

Here, it is assumed that the target road is composed of $M$ sections having the same properties 
$m(m=1, \cdots, M)$. The soundness level distribution of the target road at the regular inspection timing $t_{k}^{r}$ when the inspection/repair policy $(r, \xi) \in \Xi$ is expressed by using relative frequency $\pi_{i}^{\xi}\left(t_{k}^{r}\right)$. In addition, by using the relative frequency vector

$$
\boldsymbol{\pi}^{\xi}\left(t_{k}^{r}\right)=\left\{\pi_{1}^{\xi}\left(t_{k}^{r}\right), \cdots, \pi_{J}^{\xi}\left(t_{k}^{r}\right)\right\}
$$

the deterioration/repair process of the road can be formulated as follows:

$$
\pi_{j}^{\xi}\left(t_{k+1}^{r}\right)=\sum_{i=1}^{J} P_{i j}^{\xi}(r) \pi_{i}^{\xi}\left(t_{k}^{r}\right)
$$

The vector notation of the above equation is as follows:

$$
\boldsymbol{\pi}^{\xi}\left(t_{k+1}^{r}\right)=\boldsymbol{\pi}^{\xi}\left(t_{k}^{r}\right) \boldsymbol{P}^{\xi}(r)
$$

It is assumed that the deterioration/repair process of road pavement is repeated and the longterm steady state is reached. The stationary probability vector for the soundness level of each section is expressed by $\boldsymbol{\pi}^{r, \xi}=\left(\pi_{1}^{r, \xi}, \cdots, \pi_{J}^{r, \xi}\right)$. The stationary probability is defined as $\boldsymbol{\pi}^{r, \xi}$ that satisfies the following conditions:

$$
\begin{aligned}
& \boldsymbol{\pi}^{r, \xi}=\boldsymbol{\pi}^{r, \xi} \boldsymbol{P}^{\xi}(r) \\
& \sum_{i=1}^{J} \pi_{i}^{r, \xi}=1
\end{aligned}
$$

The set $\Xi(\bar{U})$ of inspection/repair policies $(r, \xi)$ that can keep the stationary probability $\pi_{J}^{r, \xi}$ that the soundness level $J$, which represents serviceability limit, at the road condition inspection timing, from exceeding the risk control level $\bar{U}$ is defined as follows:

$$
\Xi(\bar{U})=\left\{(r, \xi) \mid \pi_{J}^{r, \xi} \leq \bar{U}\right\}
$$

\section{(3) LCC evaluation}

This section discusses a case in which the inspection/repair policy $(r, \xi) \in \Xi(\bar{U})$ is adopted. A road administrator inspects the road condition at the timing $t_{k}^{r}(k=0,1, \cdots)$. The road condition inspection cost is expressed by $c$. If the soundness level of pavement becomes lower than the critical soundness level $i^{*}(\xi)$, the pavement is repaired. The repair cost depends on the soundness level $i$ just before the repair. The repair cost is represented by $C(i)$. The repair cost is the monotone non-decreasing function of the soundness level $i$, and satisfies the following condition:

$$
C(1) \leq C(2) \leq \cdots \leq C(J)
$$

It is assumed that the deterioration/repair process is the stationary state under the inspection/repair policy $(r, \xi)$ and soundness level is $i(i=1, \cdots, J)$ at a certain inspection timing $t_{k}^{r}$. The discounted value of the inspection/repair cost when the inspection/repair policy $(r, \xi)$ is adopted constantly after the timing $t_{k}^{r}$ (hereinafter, LCC) is represented by $V_{i}^{r, \xi}$. Since the deterioration/repair process is in the stationary state, LCC does not depend on the inspection timing $t_{k}^{r}$. If the regular inspection/repair policy $(r, \xi)$ is adopted, LCC is defined recursively as follows:

$$
\begin{aligned}
V_{i}^{r, \xi}= & c+\delta_{i}^{\xi} C(i) \\
+ & \exp (-\rho r) \sum_{j=1}^{J} P_{i j}^{\xi}(r) V_{j}^{r, \xi} \\
& (i=1, \cdots, J)
\end{aligned}
$$

where $\rho$ represents the discount rate, and $\delta_{i}^{\xi}$ denotes the dummy variable, which is defined as follows:

$$
\delta_{i}^{\xi}= \begin{cases}0 & i<i^{*}(\xi) \\ 1 & i \geq i^{*}(\xi)\end{cases}
$$

In addition, by using the stationary probability of each soundness level $\boldsymbol{\pi}^{r, \xi}=\left(\pi_{1}^{r, \xi}, \cdots, \pi_{J}^{r, \xi}\right)$ at the regular inspection timing under the inspection/repair policy $(r, \xi)$, the expected LCC in the stationary deterioration/repair process can be expressed as follows:

$$
\overline{L C C}(r, \xi)=\sum_{i=1}^{J} \pi_{i}^{r, \xi} V_{i}^{r, \xi}
$$

At this time, the optimal inspection/repair policy model for designing a pavement inspection/repair policy that minimizes the expected LCC with the specified risk control level $\bar{U}$ can be formulated as follows:

$$
\begin{aligned}
& \min _{r, \xi}\{\overline{L C C}(r, \xi)\} \\
& \text { subject to }(r, \xi) \in \Xi(\bar{U})
\end{aligned}
$$

The optimal policy, which is obtained as the optimal solution to this problem, is the conditional optimal policy with the risk control level $\bar{U}$ specified. To express it explicitly, the optimal policy is represented by $\left(r^{*}(\bar{U}), \xi^{*}(\bar{U})\right)$.

\section{EXTENDED MODEL}

\section{(1) Additional inspection/repair policy}

The basic model is focused on the decisionmaking rule in which the road condition inspection is carried out regularly and the decision is made whether to repair the pavement based on the results of the inspection. In such a rule, it may be necessary to set the risk control level so that it will be satisfied at the time of the next regular inspection. In Fig.3, if it is judged that the soundness level at the time of the regular inspection is higher than the critical soundness level 


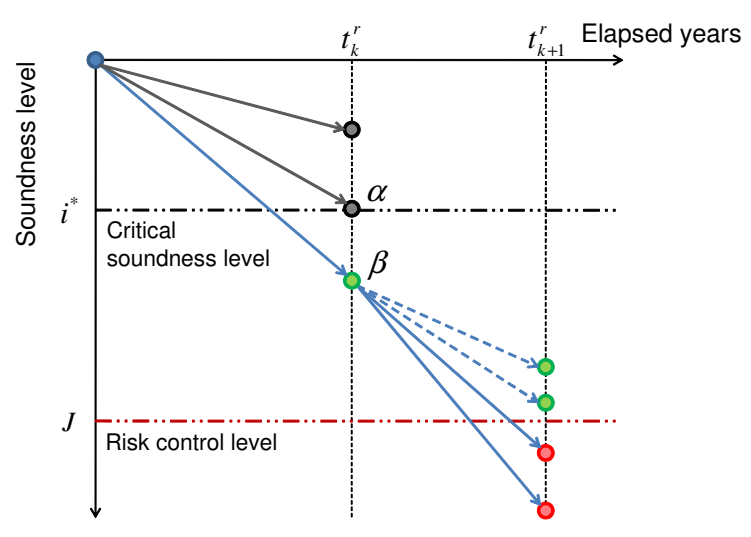

Fig.3 Risk control region.

(point $\alpha$ ), the repair of the pavement is put off until the next regular inspection. Let us discuss a case in which the soundness level is $\beta$ and repair is conducted. If repair is put off, the pavement deteriorates until the next regular inspection. This figure includes several sample paths for such a deterioration process. If deterioration progresses as represented by the solid line, the service level cannot be satisfied at the time of the next regular inspection. Accordingly, it is appropriate to repair the pavement at this regular inspection timing. If deterioration progresses as represented by the dashed line, the risk control level is satisfied at the next regular inspection timing, and so the pavement repair would augment the repair cost wastefully. The soundness level range $\left[i^{*}, J\right]$ in the figure represents the range in which repair is conducted at the present regular inspection timing in a preventive manner, because there is a possibility that the risk control level will not be satisfied at the next regular inspection timing. In this study, such a span is called the "risk control span" of soundness level. As inspection interval is longer or deterioration is more uncertain, the risk control span becomes larger. However, if soundness level is in the risk control span, the repair cost may be reduced by putting off the pavement repair until the next regular inspection, according to the deterioration state. Such inefficiency is caused by the fact that the deterioration process of pavement cannot be observed thoroughly, but can be partially observed through road condition inspection.

As a partial solution to such inefficiency, let us discuss a method of inspecting the road condition step by step. Fig. 4 shows three kinds of repair policies: (1) to conduct repair immediately (action $\left.\hat{e}_{1}\right),(2)$ to refrain from conducting repair until the next regular inspection (action $\hat{e}_{2}$ ), and (3) to carry out the follow-up inspection of the dete-

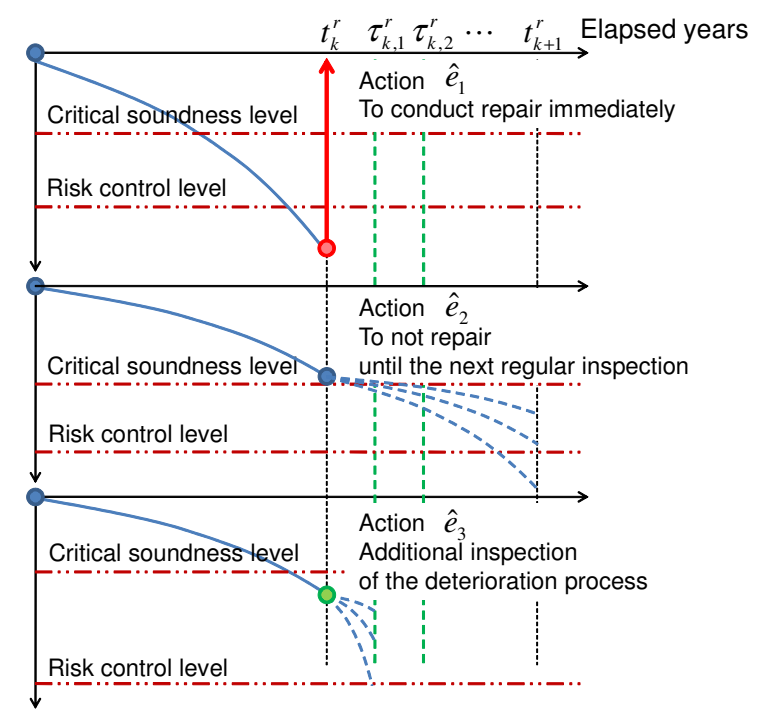

Fig.4 Additional inspection/repair policy.

rioration process and then conduct repair (action $\left.\hat{e}_{3}\right)$. By conducting the follow-up inspection of the deterioration process for the basic model, it is possible to reduce the inefficiency due to the uncertainty of the deterioration process. Needless to say, additional inspection expenses emerge for the follow-up inspection. However, the target road span of the follow-up inspection is limited, and additional inspection expenses are smaller than the expenses for regular inspection. The extended model aims at finding the regular inspection interval, repair policy, and follow-up inspection method that can minimize LCC.

\section{(2) Regular/additional inspection policy}

Like the basic model, the discrete time axis $t=0,1,2, \cdots$ with calendar time $s_{0}$ equal to the initial timing is assumed. The periodic road condition inspection (regular inspection) is conducted at the timing $t_{k}^{r}=r k$ on the discrete time axis. In addition, let us discuss a case in which the follow-up inspection is conducted during a period between the two regular inspection timings $t_{k}^{r}$ and $t_{k+1}^{r}$. Fig.4 shows that the additional inspection timing $\tau_{k, j}^{r}=t_{k}^{r}+j(j=1, \cdots, r-1)$ is set in the period $\left[t_{k}^{r}, t_{k+1}^{r}\right)$. The selectable actions $\hat{e}$ at the timing $t_{k}^{r}$ are: (1) to record the results of soundness level evaluation without conducting repair (action $\hat{e}_{1}$ ), (2) to conduct repair to recover the soundness level of pavement to 1 (action $\hat{e}_{2}$ ), and (3) to refrain from conducting repair and conduct the follow-up inspection at the timing $\tau_{k, j}^{r}(j=1, \cdots, r-1)$ (action $\left.\hat{e}_{3}\right)$. Here, the repair policy $\hat{\xi}$ at the regular inspection timing is defined with the rule $\eta^{\hat{\xi}}(i)$ for determining the soundness 
level after repair for the soundness level observed through regular inspection $h\left(t_{k}^{r}\right)=i$. The repair policy $\hat{\xi}$ can be described as follows:

$$
\begin{aligned}
& \eta^{\hat{\xi}}(i)= \begin{cases}i & \text { (in the case of action } \left.\hat{e}_{1}\right) \\
1 & \text { (in the case of action } \left.\hat{e}_{2}\right)(20) \\
i & \text { (in the case of action } \left.\hat{e}_{3}\right)\end{cases} \\
& (i=1, \cdots, J)
\end{aligned}
$$

Under the assumption that the repair policy satisfies the monotonicity condition, there are two kinds of critical soundness levels $i^{*}(\hat{\xi})$ and $i^{* *}(\hat{\xi})$, and the relations can be expressed as follows:

$$
\begin{aligned}
& \text { (when } \left.i^{*}(\hat{\xi}) \neq i^{* *}(\hat{\xi})\right) \\
& \hat{e}= \begin{cases}\hat{e}_{1} & i<i^{* *}(\hat{\xi}) \\
\hat{e}_{2} & i \geq i^{*}(\hat{\xi}) \\
\hat{e}_{3} & i^{* *}(\hat{\xi}) \leq i<i^{*}\end{cases} \\
& \text { (when } \left.i^{*}(\hat{\xi})=i^{* *}(\hat{\xi})\right) \\
& \hat{e}= \begin{cases}\hat{e}_{1} & i<i^{*}(\hat{\xi}) \\
\hat{e}_{2} & i \geq i^{*}(\hat{\xi})\end{cases} \\
& (i=1, \cdots, J)
\end{aligned}
$$

If $i^{*}(\hat{\xi})=i^{* *}(\hat{\xi})$ in the above equation, the additional inspection/repair is not conducted. In other words, repair policies $\hat{\xi}$ include the repair policies that do not include action $\hat{e}_{3}$ (composed of regular inspection and repair policies only). If action $\hat{e}_{1}$ is adopted, repair is conducted. The transitional state of soundness level before and after the above-mentioned pavement action is defined as follows like the basic model:

$$
\begin{aligned}
& q_{i j}^{\hat{\xi}}= \begin{cases}1 & \eta^{\hat{\xi}}(i)=j \\
0 & \text { otherwise }\end{cases} \\
& (i, j=1, \cdots, J)
\end{aligned}
$$

The transitional matrix with $q_{i j}^{\hat{\xi}}$ equal to the $(i, j)$ element (hereinafter, repair transitional matrix) is represented by $\boldsymbol{q}^{\hat{\xi}}$.

When action $\hat{e}_{3}$ is adopted at the timing $t_{k}^{r}$, the additional inspection is conducted at the timing $\tau_{k, j}^{r}=t_{k}^{r}+j(j=1, \cdots, r-1)$. The repair policy adopted at the additional inspection timing $\tau_{k, j}^{r}$ is defined by using the rule $\iota^{\hat{\xi}}(i)$ for determining the soundness level after repair for the soundness level $h\left(\tau_{k, j}^{r}\right)=i$ observed through additional inspection. Specifically, the repair policy $\hat{\xi}$ is defined as follows:

$$
\begin{aligned}
& { }_{\iota} \hat{\xi}_{(i)}= \begin{cases}i & \text { (in the case of action } \left.\hat{f}_{1}\right) \\
1 & \text { (in the case of action } \left.\hat{f}_{2}\right)\end{cases} \\
& \left(i=i^{* *}(\hat{\xi}), \cdots, i^{*}(\hat{\xi})-1\right)
\end{aligned}
$$

If action $\hat{f}_{2}$ is conducted at the additional inspection timing $\tau_{k, j}^{r}$, additional inspection is not carried out during the period $\left[\tau_{k, j}^{r}, t_{k+1}^{r}\right)$. It is assumed that the repair policy at the additional inspection timing satisfies the monotonicity condition. At this time, the critical soundness level $i^{\circ}(\hat{\xi})$ exists, and the additional repair rule $\hat{f}$ is expressed as follows:

$$
\begin{aligned}
& \hat{f}= \begin{cases}\hat{f}_{1} & i<i^{\circ}(\hat{\xi}) \\
\hat{f}_{2} & i \geq i^{\circ}(\hat{\xi})\end{cases} \\
& \left(i=i^{* *}(\hat{\xi}), \cdots, J\right)
\end{aligned}
$$

In addition, the set of inspection/repair policies that satisfies the monotonicity conditions (21a), (21b) and (24) is represented by $\hat{\Xi}$. If the inspection/repair policy $(r, \hat{\xi}) \in \hat{\Xi}$ is implemented and then action $\hat{f}_{2}$ is adopted according to the soundness level after inspection, repair is conducted. The transitional state of soundness level before and after such a pavement action is defined with the following expression:

$$
\begin{aligned}
& \hat{z}_{i j}^{\hat{\xi}}= \begin{cases}1 & \iota \hat{\xi} \\
0 & \text { otherwise }\end{cases} \\
& \left(i=i^{* *}(\hat{\xi}), \cdots, J ; j=1, \cdots, J\right)
\end{aligned}
$$

The transitional matrix with $\hat{z}_{i j}^{\hat{\xi}}$ equal to the $(i, j)(i, j=1, \cdots, J)$ element (hereinafter, additional repair transitional matrix) is represented by $\hat{\boldsymbol{z}}^{\hat{\xi}}$.

\section{(3) Deterioration/repair process}

Let us discuss a case in which the inspection/repair policy $(r, \hat{\xi}) \in \hat{\Xi}$ including action $\hat{e}_{3}$ is adopted and the soundness level $i(i=$ $\left.i^{* *}(\hat{\xi}), \cdots, i^{*}(\hat{\xi})-1\right)$ is observed at the regular inspection timing $t_{k}^{r}$. In this case, additional inspection is carried out unless additional inspection has not been conducted until the timing $\tau_{k, j}^{r}(j=1, \cdots, r-1)$. Here, the transitional matrix for the soundness level $i(i=$ $\left.i^{* *}(\hat{\xi}), \cdots, i^{*}(\hat{\xi})-1\right)$ is defined as follows:

$$
\boldsymbol{p}_{i^{* *}}^{i^{*}}=\left(\begin{array}{lllll}
p_{i^{* *} i^{* *}} & \cdots & p_{i^{* *} i^{*}-1} & \cdots & p_{i^{* *} J} \\
0 & \vdots & \vdots & \cdots & \vdots \\
0 & \cdots & p_{i^{*}-1 i^{*}-1} & \cdots & p_{i^{*}-1, J}
\end{array}\right)
$$

where $i^{*}=i^{*}(\hat{\xi})$ and $i^{* *}=i^{* *}(\hat{\xi})$. In addition, $(J \times J)$ matrix with the matrix $\boldsymbol{p}_{i^{* *}}^{i^{*}}$ being the partial block matrix is defined as follows:

$$
\hat{\boldsymbol{p}}^{\hat{\xi}}=\left(\begin{array}{ll}
\mathbf{0}_{i^{* *}-1}^{i^{* *}-1} & \mathbf{0}_{i^{* *}-1}^{J-i^{* *}+1} \\
\mathbf{0}_{i^{* *}-1}^{i^{*}--^{* *}} & \boldsymbol{p}_{i^{* *}}^{i^{* *}} \\
\mathbf{0}_{J-i^{* *}}^{i^{* *}}-1 & \mathbf{0}_{J-i^{*}+1}^{J-i^{* *}+1}
\end{array}\right)
$$

where $\mathbf{0}_{a}^{b}$ represents the $(a \times b)$ zero-element matrix.

Here, the additional inspection timing $\tau_{k, 1}^{r}, 1$ unit period after the regular inspection timing $t_{k}^{r}$, is focused on. Deterioration progresses from 
the regular inspection timing $t_{k}^{r}$ to the additional inspection timing $\tau_{k, 1}^{r}$, and the additional repair rule is adopted based on the results of the additional inspection at the timing $\tau_{k, 1}^{r}$. If repair is conducted in accordance with the additional repair rule, inspection is not conducted after the additional inspection timing $\tau_{k, 1}^{r}$ and regular inspection is carried out at the regular inspection timing $t_{k+1}^{r}$. On the other hand, if repair is not conducted, deterioration progresses until the additional inspection timing $\tau_{k, 2}^{r}$. Then, the same procedures are repeated, and additional inspection is continued until the timing $\tau_{r-1}^{r}$ unless repair is conducted. It is noteworthy that the probability $\tilde{p}_{i k}(j)$ that the soundness level $i$ changes to the soundness level $k, j$ period later can be defined as follows:

$$
\tilde{p}_{i k}(j)=\sum_{l=1}^{k-1} p_{i l}(j-1) p_{l k}
$$

In addition, the transitional matrix with the transitional probability $\tilde{p}_{i k}(j)$ equal to the $(i, k)$ element is represented by $\tilde{\boldsymbol{p}}(j)$. The deterioration/inspection process under the additional inspection/repair policy $\hat{\xi}$ can be expressed as follows, by using the transitional matrices (2), (25), (27) and (28):

$$
\hat{\boldsymbol{p}}^{\hat{\xi}}(r)=\sum_{j=1}^{r-1} \hat{\boldsymbol{p}}^{\hat{\xi}} \tilde{\boldsymbol{p}}(j-1) \hat{\boldsymbol{z}}^{\hat{\xi}}\{\boldsymbol{p}\}^{r-j}
$$

where $\tilde{\boldsymbol{p}}(0)$ is equal to the unit matrix $\tilde{\boldsymbol{I}}$. The $(i, j)$ element of the matrix $\hat{\boldsymbol{p}}^{\hat{\xi}}(r)$ is denoted by $\hat{p}_{i j}^{\hat{\xi}}(r)$. At this time, the transitional probability representing the deterioration/repair process under the inspection/repair policy $(r, \hat{\xi}) \in \hat{\Xi}$ in the period $\left[t_{k}^{r}, t_{k+1}^{r}\right)$ can be expressed by the $(J \times J)$ matrix $\hat{\boldsymbol{P}}^{\hat{\xi}}(r)$ with the $(i, j)$ element as follows:

$$
\begin{aligned}
& \text { (when } i^{*}(\hat{\xi}) \neq i^{* *}(\hat{\xi}) \text { ) } \\
& \hat{P}_{i j}^{\hat{\xi}}(r)=\left\{\begin{array}{l}
P_{i j}^{\hat{\xi}}(r) \quad<i^{* *}(\hat{\xi}) \\
P_{i j}^{\hat{\xi}}(r) \quad i \geq i^{*}(\hat{\xi}) \\
\hat{p}_{i j}^{\hat{\xi}}(r) \quad i^{* *}(\hat{\xi}) \leq i<i^{*}(\hat{\xi})
\end{array}\right. \\
& \text { (when } i^{*}(\hat{\xi})=i^{* *}(\hat{\xi}) \text { ) } \\
& \hat{P}_{i j}^{\hat{\xi}}(r)=\left\{\begin{array}{cc}
P_{i j}^{\hat{\xi}}(r) & i<i^{*}(\hat{\xi}) \\
P_{i j}^{\hat{\xi}}(r) & i \geq i^{*}(\hat{\xi})
\end{array}\right.
\end{aligned}
$$

where $P_{i j}^{\hat{\xi}}(r)$ is defined with Equation (9). The soundness level distribution for the target road at the regular inspection timing $t_{k}^{r}$ when the inspection/repair policy $(r, \hat{\xi}) \in \hat{\Xi}$ is adopted is expressed by using the relative frequency $\pi_{i}^{\hat{\xi}}\left(t_{k}^{r}\right)$. In addition, by using the relative frequency vector:

$$
\boldsymbol{\pi}^{\hat{\xi}}\left(t_{k}^{r}\right)=\left\{\pi_{1}^{\hat{\xi}}\left(t_{k}^{r}\right), \cdots, \pi_{J}^{\hat{\xi}}\left(t_{k}^{r}\right)\right\}
$$

the deterioration/repair process of the target road can be formulated as follows:

$$
\pi_{j}^{\hat{\xi}}\left(t_{k+1}^{r}\right)=\sum_{i=1}^{J} \hat{P}_{i j}^{\hat{\xi}}(r) \pi_{i}^{\hat{\xi}}\left(t_{k}^{r}\right)
$$

The vector notation of the above equation is as follows:

$$
\boldsymbol{\pi}^{\hat{\xi}}\left(t_{k+1}^{r}\right)=\boldsymbol{\pi}^{\hat{\xi}}\left(t_{k}^{r}\right) \hat{\boldsymbol{P}}^{\hat{\xi}}(r)
$$

It is assumed that the deterioration/repair process of road pavement is repeated and the longterm stationary state is reached. The stationary probability vector for the soundness level of each section is represented by $\boldsymbol{\pi}^{r, \hat{\xi}}=\left(\pi_{1}^{r, \hat{\xi}}, \cdots, \pi_{J}^{r, \hat{\xi}}\right)$. The stationary probability is defined as $\boldsymbol{\pi}^{r, \hat{\xi}}$ that satisfies the following relation:

$$
\boldsymbol{\pi}^{r, \hat{\xi}}=\boldsymbol{\pi}^{r, \hat{\xi}} \hat{\boldsymbol{P}}^{\hat{\xi}}(r)
$$

The set $\hat{\Xi}(\bar{U})$ of the inspection/repair policies $(r, \hat{\xi})$ that can keep the stationary probability $\pi_{J}^{r, \hat{\xi}}$ that the soundness level $J$, which is serviceability limit, is observed at the road condition inspection timing lower than the risk control level $\bar{U}$ is defined as follows:

$$
\hat{\Xi}(\bar{U})=\left\{(r, \hat{\xi}) \mid \pi_{J}^{r, \hat{\xi}} \leq \bar{U}\right\}
$$

\section{(4) LCC evaluation}

Let us discuss a case in which the inspection/repair policy $(r, \hat{\xi}) \in \hat{\Xi}(\bar{U})$ is adopted. A road administrator inspects the road condition at the timing $t_{k}^{r}(k=0,1, \cdots)$. The road condition inspection cost is represented by $c$. If the soundness level of pavement is higher than the critical soundness level $i^{*}(\hat{\xi})$ at the road condition inspection timing, the pavement is repaired. In this case, the repair cost $C(i)$ emerges according to the soundness level $i$. If soundness level satisfies the condition: $i^{* *}(\hat{\xi}) \leq i<i^{*}(\hat{\xi})$, the additional inspection/repair policy is adopted from the inspection timing. The additional inspection/repair cost $C^{r, \hat{\xi}}(i)$ that emerges until the next inspection timing can be expressed as follows:

$$
\begin{aligned}
C^{r, \hat{\xi}}(i) & =\sum_{j=1}^{r-1} \sum_{k=i}^{J} \exp (-\rho j) \tilde{p}_{i k}(j) z_{k 1} C(k) \\
& +\sum_{j=1}^{r-1} \sum_{k=i}^{i^{\circ}-1} \exp (-\rho j) p_{i k}(j-1) w
\end{aligned}
$$

where $w$ represents the additional inspection cost, $i^{\circ}=i^{\circ}(\hat{\xi}), p_{i k}(j)$ is the probability that soundness level changes from $i$ to $k$ from the timing $t_{k}^{r}$ 
to the $j$-th period, and the $(i, k)$ element of the matrix $\boldsymbol{p}(j) . \tilde{p}_{i k}(j)$ denotes the probability that soundness level changes to $k$ in the $j$-th period, and is expressed by Equation (28).

Next, it is assumed that the deterioration/repair process is stationary under the inspection/repair policy $(r, \hat{\xi})$ and soundness level turns out to be $i(i=1, \cdots, J)$ at a regular inspection timing $t_{k}^{r}$. The discounted present value of the inspection/repair cost (hereinafter, LCC) when the inspection/repair policy $(r, \hat{\xi})$ is implemented constantly after the timing $t_{k}^{r}$ is represented by $V_{i}^{r, \hat{\xi}}$. Since the deterioration/repair process is stationary, LCC does not depend on the regular inspection timing $t_{k}^{r}$. When the inspection/repair policy $(r, \hat{\xi})$ is adopted, LCC is defined recursively as follows:

$$
\begin{gathered}
V_{i}^{r, \hat{\xi}}=c+\delta_{i}^{\hat{\xi}} C(i)+\hat{\delta}_{i}^{\hat{\xi}} C^{r, \hat{\xi}}(i) \\
+\exp (-\rho r) \sum_{j=1}^{J} \hat{P}_{i j}^{\hat{\xi}}(r) V_{j}^{r, \hat{\xi}} \\
(i=1, \cdots, J)
\end{gathered}
$$

In Equation (37), the first and second terms of the right-hand side represent the regular inspection and repair costs in the $k$-th period, respectively, the third term represents the additional inspection/repair cost, and the fourth term represents the present value of LCC after the $k+1$-th period. Here, $\delta_{i}^{\hat{\xi}}, \hat{\delta}_{i}^{\hat{\xi}}$ are dummy variables defined as follows:

$$
\begin{aligned}
& \delta_{i}^{\hat{\xi}}= \begin{cases}0 & i \leq i^{*}(\hat{\xi}) \\
1 & i>i^{*}(\hat{\xi})\end{cases} \\
& \hat{\delta}_{i}^{\hat{\xi}}= \begin{cases}1 & i^{* *}(\hat{\xi}) \leq i<i^{*}(\hat{\xi}) \\
0 & \text { (when } \left.i^{*}(\hat{\xi}) \neq i^{* *}(\hat{\xi})\right)\end{cases}
\end{aligned}
$$

By using the stationary probability $\boldsymbol{\pi}^{r, \hat{\xi}}=$ $\left(\pi_{1}^{r, \hat{\xi}}, \cdots, \pi_{J}^{r, \hat{\xi}}\right)$ of each soundness level $\pi$ at the regular inspection timing under the inspection/repair policy $(r, \hat{\xi})$, the expected LCC in the stationary deterioration/repair process can be expressed as follows:

$$
\overline{L C C}(r, \hat{\xi})=\sum_{i=1}^{J} \pi_{i}^{r, \hat{\xi}} V_{i}^{r, \hat{\xi}}
$$

At this time, the optimal inspection/repair policy model for designing the pavement inspection/repair policy that minimizes the expected LCC with the risk control level $\bar{U}$ specified can be formulated as follows:

$$
\begin{aligned}
& \min _{r, \hat{\xi}}\{\overline{L C C}(r, \hat{\xi})\} \\
& \text { subject to }(r, \hat{\xi}) \in \hat{\Xi}(\bar{U})
\end{aligned}
$$

The optimal policy, which is the optimal solution to this problem, is the conditional optimal policy with the risk control level $\bar{U}$ specified, and to express it explicitly, the optimal policy is represented by $\left(r^{* *}(\bar{U}), \hat{\xi}^{* *}(\bar{U})\right)$.

\section{BENEFITS OF INSPECTION AND THE METHOD FOR SOLV- ING THE MODEL}

\section{(1) Method for evaluating the benefits of inspection}

By inspecting the road condition, it is possible to obtain the data on the soundness level of pavement in each road span. Road administrators decide whether to repair each road span based on the data of soundness level, by using the condition-dependent rule that depends on the soundness level of pavement. If the road condition is not inspected, the data on the damage to pavement cannot be obtained, and so we have no choice but to repair pavement periodically regardless of the damage state of pavement. Pavement is repaired based on the time-dependent rule that is implemented at predetermined intervals. The economic benefit of the periodic road condition inspection can be defined by using the decrease in LCC when the time-dependent rule is changed to the condition-dependent rule. By inspecting the road condition, road administrators may opt (real option) to repair the roads or not. In this light, the economic value of the road condition inspection can be interpreted as the option value for rationalizing the decision making regarding the timing of pavement repair.

To evaluate the economic benefit of the road condition inspection, let us estimate LCC under the time-dependent rule, which is the target of comparison. Like the basic model, the discrete time axis $t=0,1,2, \cdots$ is considered. It is assumed that pavement has been repaired at the timing $t$. The deterioration process during a period from the previous repair timing $t$ to the timing $t+r$ can be expressed by the transitional probability matrix $\boldsymbol{p}(r)$ shown in Equation (4). The probability that soundness level changes to the soundness level $J$, which represents serviceability limit, at the timing $t+r$ can be represented by $p_{1 J}(r)$. In the case where the time-dependent rule is adopted, if the risk control level is set at $\bar{U}$, the optimal repair interval $r^{\circ}$ can be defined as follows:

$$
r^{\circ}=\max _{r}\left\{r \mid p_{1 J}(r) \leq \bar{U}(r=1,2, \cdots)\right\}
$$

In addition, the LCC under the time-dependent 
rule with repair interval equal to $r^{\circ}$ is defined recursively as follows:

$$
V^{r^{\circ}}=\sum_{k=1}^{J} p_{1 k}\left(r^{\circ}\right) C(k)+\exp \left(-\rho r^{\circ}\right) V^{r^{\circ}}
$$

With the above equation, LCC $V^{r^{\circ}}$ under the time-dependent rule is expressed as follows:

$$
V^{r^{\circ}}=\frac{\sum_{k=1}^{J} p_{1 k}\left(r^{\circ}\right) C(k)}{1-\exp \left(-\rho r^{\circ}\right)}
$$

On the other hand, LCC under the conditiondependent rule is expressed as follows, based on Equation (18):

$$
\overline{L C C}\left(r^{*}, \xi^{*}\right)=\sum_{i=1}^{J} \pi_{i}^{r^{*}, \xi^{*}} V_{i}^{r^{*}, \xi^{*}}
$$

Accordingly, the net benefit $B$ of the shift from the time-dependent rule to the conditiondependent rule is expressed as follows:

$$
B=V^{r^{\circ}}-\overline{L C C}\left(r^{*}, \xi^{*}\right)
$$

In addition, to evaluate economic benefit (net benefit) per regular inspection, the economic benefit $B$ is expressed by the sequence of equivalent benefits. At this time, the average net benefit $\Delta b^{*}$ per regular inspection can be defined as follows by using $b^{*}$ that satisfies the following relation:

$$
\begin{gathered}
B=\frac{b^{*}}{1-\exp (-\rho)} \\
\Delta b^{*}=\sum_{k=0}^{r^{*}-1} \exp (-\rho k) b^{*}
\end{gathered}
$$

Next, the economic benefit of additional inspection is defined by using the decrease in LCC when the situation in which the road condition is inspected regularly is shifted to the situation in which additional inspection is carried out. If additional inspection is conducted, the expected LCC in the stationary deterioration/repair process can be expressed as follows:

$$
\overline{L C C}\left(r^{* *}, \hat{\xi}^{* *}\right)=\sum_{i=1}^{J} \pi_{i}^{r^{* *}, \hat{\xi}^{* *}} V_{i}^{r^{* *}, \hat{\xi}^{* *}}
$$

Accordingly, the economic benefit $\bar{B}$ of additional inspection can be defined as follows:

$$
\bar{B}=\overline{L C C}\left(r^{*}, \xi^{*}\right)-\overline{L C C}\left(r^{* *}, \hat{\xi}^{* *}\right)
$$

In addition, the average net benefit of the entire group of additional inspections after a single regular inspection (maximum $r^{* *}-1$ additional inspection) can be formulated, by using $b^{* *}$ that satisfies the following relation:

$$
\begin{gathered}
\bar{B}=\frac{b^{* *}}{1-\exp (-\rho)} \\
\Delta b^{* *}=\sum_{k=0}^{r^{* *}-1} \exp (-\rho k) b^{* *}
\end{gathered}
$$

\section{(2) Solution}

Let us propose a method for designing the optimal inspection/repair policy of the basic model. It is first assumed that the regular inspection/repair policy $(r, \xi)$ is given. In this case, LCC is recursively defined as follows:

$$
\begin{aligned}
V_{i}^{r, \xi}= & c+\delta_{i}^{\xi} C(i) \\
+ & \exp (-\rho r) \sum_{j=1}^{J} P_{i j}^{\xi}(r) V_{j}^{r, \xi} \\
& (i=1, \cdots, J)
\end{aligned}
$$

By solving the simultaneous Equation (52), it is possible to obtain $V_{i}^{r, \xi}(i=1, \cdots, J)$. By calculating $V_{i}^{r, \xi}(i=1, \cdots, J)$ for every $(r, \xi) \in$ $\Xi$, it is possible to obtain the optimal solution $\left(r^{*}(\bar{U}), \xi^{*}(\bar{U})\right)$ to the problems (19a) and (19b). Considering this, the solving method of the basic model can be summarized as shown in Fig.5, that is,

1) step 1 Set $(r, \xi)$.

2) step 2 Obtain the transitional probability $\boldsymbol{P}^{\xi}(r)$.

3) step 3 Discuss whether $\pi_{J}^{r, \xi} \leq \bar{U}$ is satisfied. If it is not satisfied, proceed to Step 7. If satisfied, proceed to Step 4.

4) step 4 Solve the simultaneous Equation (52), to obtain $V_{i}^{r, \xi}(i=1, \cdots, J)$.

5) step $\mathbf{5}$ Obtain the stationary probability $\boldsymbol{\pi}^{r, \xi}$ with Equations (14a) and (14b).

6) step 6 Obtain $\overline{L C C}\left(r^{*}, \xi^{*}\right)$ with Equation (44)

7) step 7 If search has not been finished for every $(r, \xi)$, set new $(r, \xi)$, and return to Step 2 . If search has been finished for every $(r, \xi)$, obtain $\left(r^{*}, \xi^{*}\right)$ that minimizes $\overline{L C C}\left(r^{*}, \xi^{*}\right)$.

Next, let us propose a method for designing the optimal inspection/repair policy of the extended model. If the inspection/repair policy $(r, \hat{\xi})$ is adopted, the LCC is defined recursively as follows:

$$
\begin{aligned}
V_{i}^{r, \hat{\xi}}= & c+\delta_{i}^{\hat{\xi}} C(i)+\hat{\delta}_{i}^{\hat{\xi}} C^{r, \hat{\xi}}(i) \\
+ & \exp (-\rho r) \sum_{j=1}^{J} P_{i j}^{\hat{\xi}}(r) V_{j}^{r, \hat{\xi}} \\
& (i=1, \cdots, J)
\end{aligned}
$$

By solving the simultaneous Equation (53), it is possible to solve $V_{i}^{r, \xi}(i=1, \cdots, J)$. The solving method of the extended model is the same as that for the basic model, and can be summarized as follows:

1) step $1 \quad$ Set $(r, \hat{\xi})$.

2) step 2 Obtain the transitional probability $\hat{\boldsymbol{P}}^{\hat{\xi}}(r)$. 


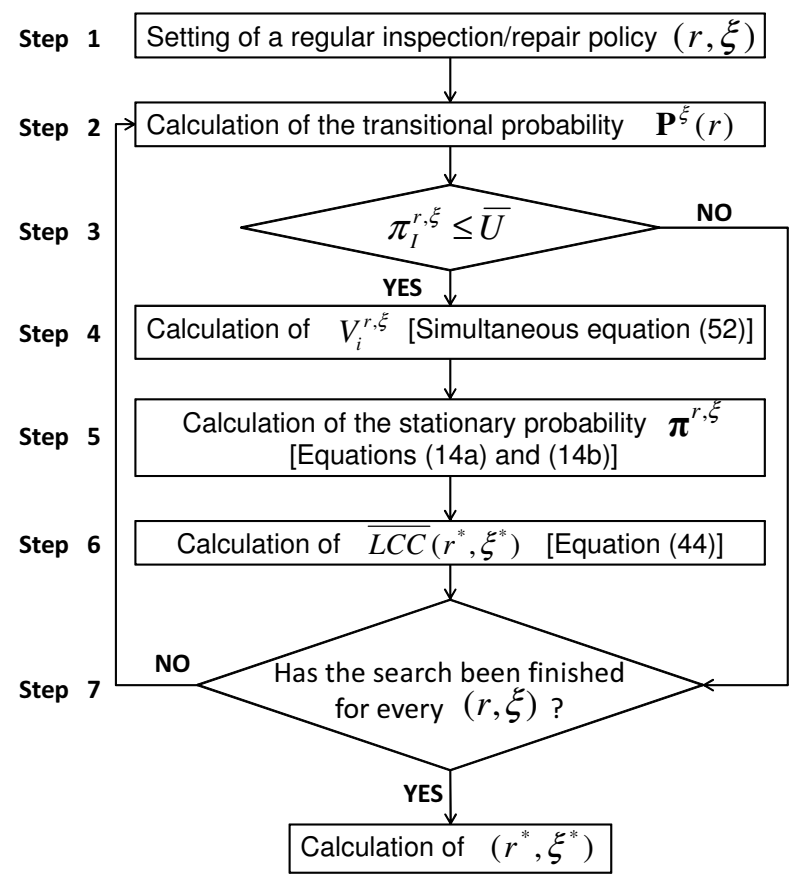

Fig.5 Solving method of the optimal inspection/repair model.

3) step 3 Discuss whether $\pi_{J}^{r, \hat{\xi}} \leq \bar{U}$ is satisfied. If it is not satisfied, proceed to Step 7 . If satisfied, proceed to Step 4.

4) step 4 Solve the simultaneous Equation (53), to obtain $V_{i}^{r, \hat{\xi}}(i=1, \cdots, J)$.

$5)$ step 5 Obtain the stationary probability $\boldsymbol{\pi}^{r, \hat{\xi}}$ with Equations (14a) and (14b).

6) step 6 Obtain $\overline{L C C}\left(r^{* *}, \hat{\xi}^{* *}\right)$ with Equation (48).

7) step 7 If search has not been finished for every $(r, \hat{\xi})$, set new $(r, \hat{\xi})$, and return to Step 2 . If search has been finished for every $(r, \hat{\xi})$, obtain $\left(r^{* *}, \hat{\xi}^{* *}\right)$ that minimizes $\overline{L C C}\left(r^{* *}, \hat{\xi}^{* *}\right)$.

\section{APPLICATION CASES}

\section{(1) Outline of application cases}

In this study, the authors focus on the pavement management for expressways as an application case, and discuss the problem of inspecting the soundness level of pavement regularly through road condition inspection using a road condition monitoring vehicle. The target road span is managed by NEXCO, and has 6 lanes and a total length of about $300 \mathrm{~km}$. It has been 35 years since it was first used, and the traffic volume of largesized vehicles is about 6,800 per day. The pavement of this span is of the drainage type, and about $95 \%$ of this span is earthwork.

The soundness level of pavement is evaluated for each of the three types of damages: (1) cracking, (2) rutting, and (3) IRI. In the road condition inspection, the data for the three items of soundness level are obtained at the same time while defining a $100 \mathrm{~m}$ span as a basic unit, and the soundness level of pavement is evaluated. Based on the results of this road condition inspection, the parts to be repaired are selected. In detail, a relatively long road span is inspected simultaneously, and the damage state of pavement is evaluated for each $100 \mathrm{~m}$ span, to select the spans to be repaired. The reference value for repair is predetermined for each of the three kinds of damages, and if either of the values of the three kinds of damages in a certain road span reaches the reference value for repair, the road span is considered as a span to be repaired. In this case, expressway pavement is repaired using the cutting overlay method as the basic repair method, and it is assumed that the same repair method is adopted regardless of the progress of damage. Accordingly, the repair cost depends on the length of only the pavement to be repaired.

As the condition for calculating LCC, the road condition inspection cost was set at 20,000 yen $/ \mathrm{km}$, with reference to the standards for calculation for inspection, etc. in 2009 (East, Central, and West Nippon Expressway Company). It was assumed that the drainage pavement had a density of $1.98 \mathrm{t} / \mathrm{m}^{3}$ and a $4 \mathrm{~cm}$-thick surface layer, and the cost was set at 2,125 yen $/ \mathrm{m}^{2}$ with reference to the above-mentioned standard for estimation and the table of construction costs and construction machine rents, etc. in 2009. Under the assumption that the unit span was $100 \mathrm{~m}$ in length and $3.5 \mathrm{~m}$ in width, the repair cost per unit span was 744,000 yen, and the inspection cost per unit span was 2,000 yen.

\section{(2) Development of a Markov deteriora- tion model}

Assuming that each type of damage progresses independently, the deterioration process of each type is expressed with a Markov deterioration model, and the optimal inspection interval is obtained by using the optimal inspection/repair model. Then, the damage type with the shortest inspection interval is focused on, to design the optimal inspection/repair policy. In detail, it is necessary to develop a deterioration prediction model that takes into account all of the three types of damages, focus on the most deteriorated damage type, and design the optimal inspection/repair policy. The authors have proposed a competitive deterioration hazard model ${ }^{19)}$ that takes into account the three kinds of damages. 
Table 1 Soundness level.

\begin{tabular}{c|ccc}
\hline Level & Rutting:Ru $(\mathrm{mm})$ & Cracking:Cr(\%) & $I R I(\mathrm{~mm} / \mathrm{m})$ \\
\hline 1 & $R u \leq 5$ & $C r=0$ & $I R I \leq 1.0$ \\
2 & $5<R u \leq 9$ & $0<C r \leq 1.0$ & $1.0<I R I \leq 1.5$ \\
3 & $9<R u \leq 13$ & $1.0<C r \leq 2.5$ & $1.5<I R I \leq 2.0$ \\
4 & $13<R u \leq 17$ & $2.5<C r \leq 5.0$ & $2.0<I R I \leq 2.5$ \\
5 & $17<R u \leq 21$ & $5.0<C r \leq 10.0$ & $2.5<I R I \leq 3.0$ \\
6 & $21<R u \leq 25$ & $10.0<C r \leq 20.0$ & $3.0<I R I \leq 3.5$ \\
7 & $25<R u$ & $20.0<C r$ & $3.5<I R I$ \\
\hline
\end{tabular}

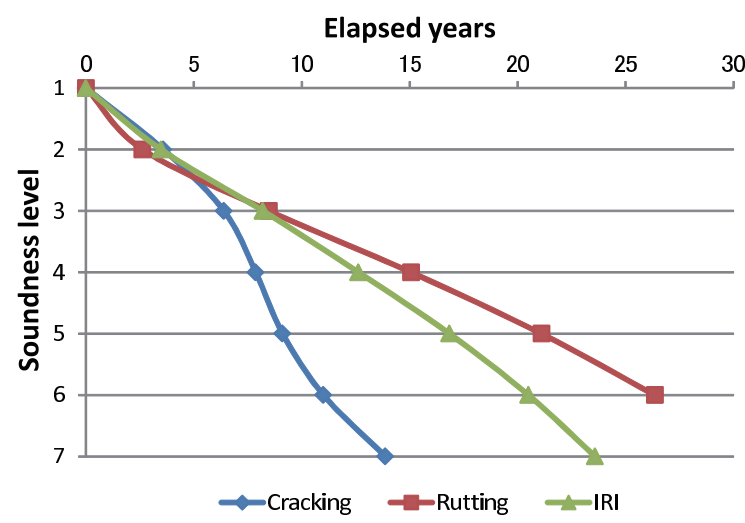

Fig.6 Performance curve.

However, it is known that in case where the competitive deterioration hazard model is used, even if the deterioration for each type of damage has Markov property, the Markov transitional probability that takes into account the three kinds of damage types does not have Markov property. Accordingly, the process of designing the optimal inspection/repair policy based on the optimal inspection/repair model is extremely complex and impractical. Here, let us focus on the most rapid damage type, and design the most desirable inspection/repair policy. In this study, the basic unit for repair is $100 \mathrm{~m}$. In actual scenarios of maintenance and management, the road spans adjacent to the pavement to be repaired are considered, and the adjoining spans are repaired at once in many cases. Such a policy of putting together some spans is not adopted, and repair is conducted with a $100 \mathrm{~m}$ unit span.

The authors have estimated a Markov deterioration model for cracking, rutting, and IRI, based on the data of the road condition inspection of expressways throughout Japan ${ }^{20)}$. In this application case, the optimal interval of the road condition inspection is obtained by using the Markov deterioration model estimated in the previous study ${ }^{20)}$. Fig. 6 shows the performance curves of cracking, rutting, and IRI plotted based on the estimation results of the Markov deteriora-

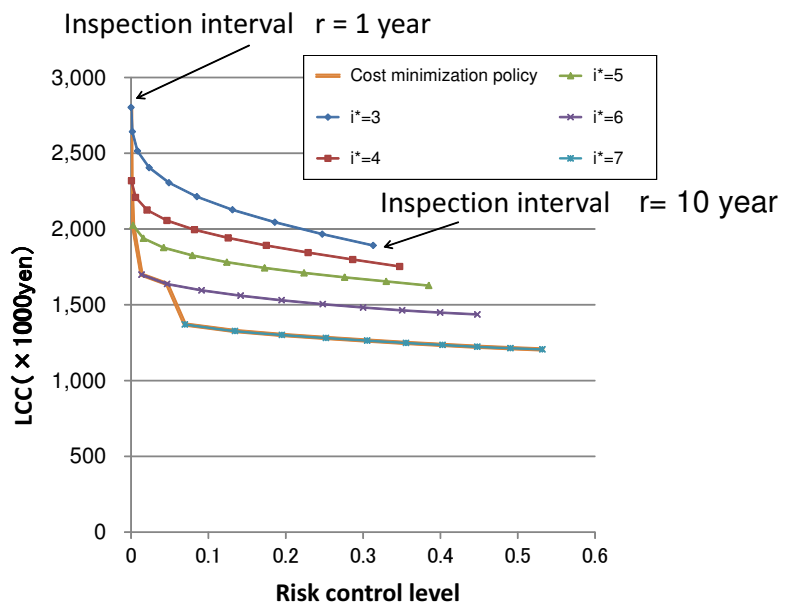

Fig.7 Relation between risk control level and LCC (basic model).

tion model. The vertical axis of this figure represents the soundness level for each type of damage. Table 1 shows the relation between soundness level and the road condition for each type of damage. The most damaged state is expressed by $J=7$. As shown in Fig.6, the road condition data for rutting did not include a case in which $J=7$. These performance curves indicate that the deterioration speed for cracking is the highest. Accordingly, let us focus on cracking as the representative road damage type for discussing the desirable inspection/repair policy.

\section{(3) Analysis results of the basic model}

Fig. 7 shows the results of the analysis of the relation between the risk control level and LCC obtained by using the basic model. In this figure, for the cases in which the critical soundness level $i^{*}(\xi)$ is set from $i^{*}(\xi)=3$ to $i^{*}(\xi)=7$, the interval of the road condition inspection is changed from one to ten years by years, to clarify the relation between LCC and risk control level. Regardless of the critical soundness level $i^{*}(\xi)$, it is possible to find the trade-off relation between LCC and risk control level. The figure shows the curves for five critical soundness levels $i^{*}(\xi)$, and the envelope curve (risk-cost control curve) drawn 
based on the minimum LCC policy under the condition that the specified risk control level is satisfied. The gradient of the risk-cost control curve is low in the region where risk control level is over 0.1 (right side of the figure). This indicates that the decrease in inspection frequency has a small effect on the reduction in LCC, while the decrease in inspection frequency augments the risk of deterioration significantly. On the other hand, when risk control level is increased to over 0.07 , inspection frequency increases and LCC augments. In the case where risk control level is over 0.07 , by setting the critical soundness level $i$ at $i^{*}(\xi)=7$, it is possible to minimize LCC. Thus, it is most appropriate to conduct repair when the deterioration of pavement has reached the control level. However, in this study, inspection interval was set in units of years, and so there was no feasible solution for making risk control level less than 0.07 . In the case where large-scale inspection interval is set in units of years, in order to make risk control level less than 0.07 , it is necessary to set critical soundness level at less than $i^{*}(\xi)=6$. In case risk control level is less than 0.07 as a target value, preventive maintenance policy has to be adopted. In case ex-post maintenance policy is adopted, to set risk control level less than 0.07, it is necessary to set inspection interval at less than one year. However, conducting large-scale inspection several times in the same fiscal year is not practical and augments LCC significantly. The following section analyzes the effects of the additional inspection/repair policy by using the extended model.

\section{(4) Analysis results of the extended model}

In the extended model, two critical soundness levels $i^{*}(\hat{\xi})$ and $i^{* *}(\hat{\xi})$ are used, and the following three repair policies are discussed: (1) to conduct repair immediately $\left(i \geq i^{*}(\hat{\xi})\right)$, (2) to not conduct repair until the next regular inspection $\left(i<i^{* *}(\hat{\xi})\right)$, and (3) to conduct additional inspection of the deterioration process, and repair it if necessary $\left(i^{* *}(\hat{\xi}) \leq i<i^{*}(\hat{\xi})\right)$. If $i^{*}(\hat{\xi})=i^{* *}(\hat{\xi})$, the additional inspection/repair policy is not implemented, and the following two repair policies are discussed: (1) to repair it immediately $\left(i \geq i^{*}(\hat{\xi})\right)$, and (2) to not conduct inspection or repair until the next regular inspection $\left(i<i^{*}(\hat{\xi})\right)$. In addition, the following two cases are specified for analysis: (1) the case in which the critical soundness level $i^{*}(\hat{\xi})$ for immediate repair is set at 7 and the critical soundness level for additional inspection/repair $i^{* *}(\hat{\xi})$

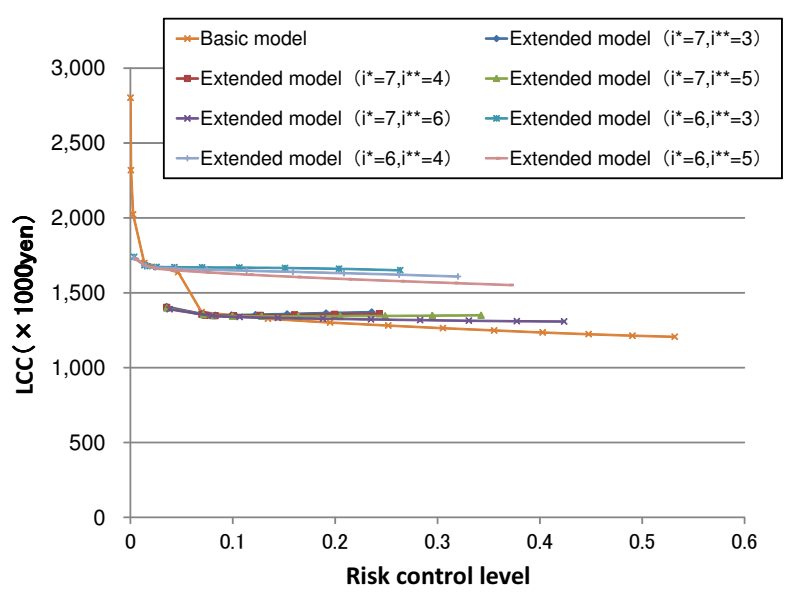

Fig.8 Risk-cost control curve (extended model).

is changed between 3 and 6 , and (2) the case in which $i^{*}(\hat{\xi})$ is set at 6 , and $i^{* *}(\hat{\xi})$ is changed between 3 and 5. Fig. 8 shows the results of the analysis of the relation between risk control level and LCC for these two cases. This figure includes the cost minimizing policy obtained with the basic model. The cycle of the road condition inspection under the additional inspection/repair policy changes from one to ten years by years like the basic model. When the interval is one year, additional inspection is conducted six months later. In the case of the extended model, as risk control level increases, it becomes necessary to increase the frequency of the road condition inspection, and LCC augments. However, the trade-off relation between risk control level and LCC in the extended model is flatter than that in the basic model. Thus, even when risk control level is increased, by adopting the meticulous additional inspection/repair policy, it is possible to curb the increase in LCC. If the risk control level is higher than 0.04 , by setting the critical soundness level $i^{*}(\hat{\xi})$ at 7 (adopting the ex-post maintenance policy), it is possible to minimize LCC. If the risk control level is equal to 0.07 , the road condition inspection interval $r^{* *}$ is equal to 3 . In the case of the basic model, to achieve the same risk control level 0.07 , it is necessary to inspect the road condition every year. By conducting additional inspection/repair, it becomes possible to extend the optimal road condition inspection interval from one to three years. Like the case of the basic model, to set risk control level at 0.04 or lower, it is necessary to set the critical soundness level $i^{*}(\hat{\xi})$ at 6 and implement the preventive maintenance, but LCC augments significantly.

Fig.9 shows the results of the analysis of the relation between risk control level and LCC when 


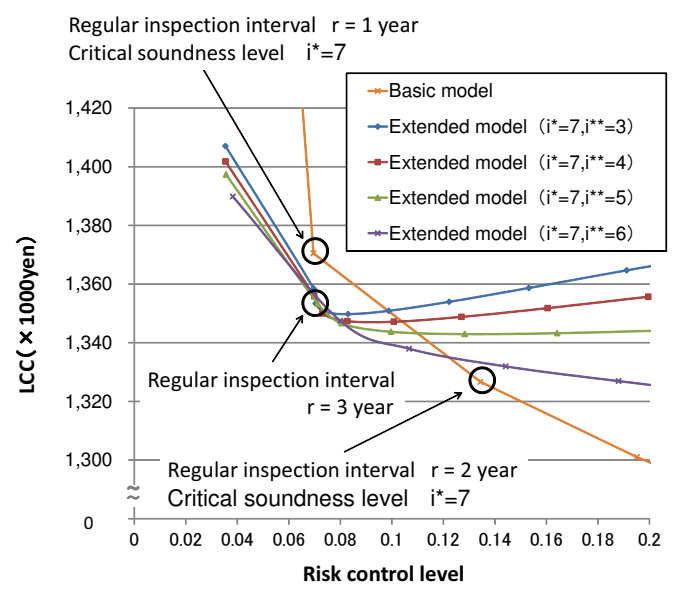

Fig.9 Risk-cost control curve (extended model: $\left.i^{*}(\hat{\xi})=7\right)$.

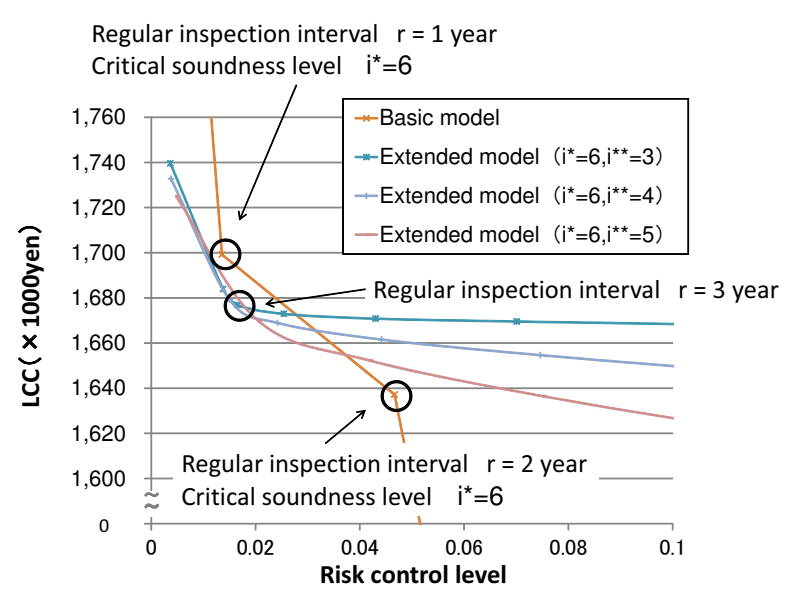

Fig.10 Risk-cost control curve (extended model: $\left.i^{*}(\hat{\xi})=6\right)$.

risk control level is 0.2 or lower, under the assumption that the critical soundness level $i^{*}(\hat{\xi})$ is set at 7 . In the case of the basic model, to keep risk control level 0.1 or lower, it is necessary to inspect the road condition at least once per year. In the case of the extended model, it is possible to keep the risk control level at 0.1 or lower, by inspecting the road condition at four-year intervals and conducting additional inspection/repair every year. In this case, the critical soundness level $i^{* *}(\hat{\xi})$ is set at 3 to 5 . Compared with the basic model, there is a small effect of cost reduction. In many cases of pavement management for expressways, the road condition is inspected once per three years. However, it was found that when the ex-post maintenance policy was adopted, it was impossible to keep risk control level about 0.07 . In this case, by implementing the additional inspection/repair policy every year, it becomes possible to keep risk control level about 0.04. Fig.10

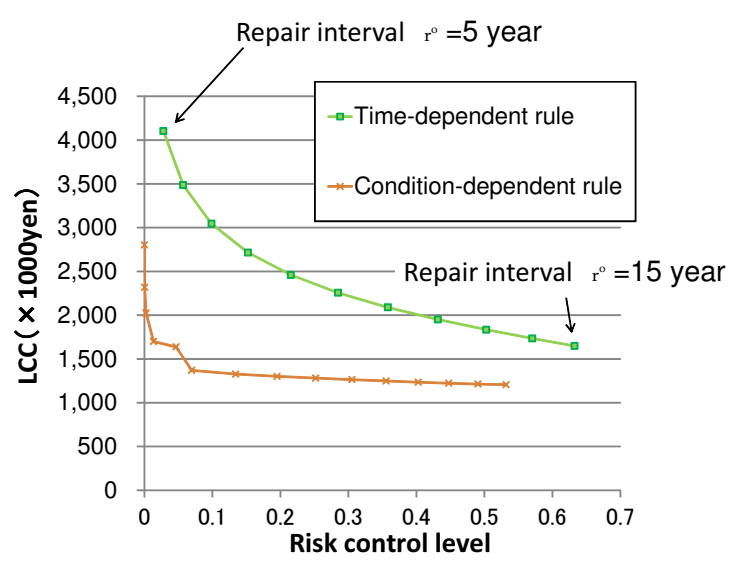

Fig.11 Risk-cost control curve (time-dependent rule).

enlarges the region where risk control level is 0.02 to 0.06 when $i^{*}(\hat{\xi})=6$. In the case of the basic model, by implementing the preventive maintenance policy $i^{*}(\hat{\xi})=6$, it is possible to keep risk control level less than 0.04. However, the comparison between Fig.9 and Fig.10 indicates that LCC augments when the critical soundness level $i^{*}$ is changed from 7 to 6 . This is because the inspection cost is smaller than the repair cost, and to achieve a risk control level of 0.04 , it is better to adopt the ex-post maintenance policy $i^{*}(\hat{\xi})=7$ and conduct additional inspection than to implement the preventive maintenance $i^{*}(\hat{\xi})=6$, from the viewpoint of the minimization of LCC.

\section{(5) Analysis results of the benefits of in- spection}

Next, let us analyze the economic benefits (net benefits) of the road condition inspection and the additional inspection/repair policy. Fig.11 shows the risk-cost control curve when the time-dependent rule calculated with Equations (41),(42) and (43) is adopted. The figure also includes the risk-cost control curve for the basic model as the condition-dependent rule. The repair interval $r^{\circ}$ of the time-dependent rule is changed from 5 to 15 years by years. In the case of the time-dependent rule, the trade-off relation between LCC and risk control level is clearly indicated like the case of the condition-dependent rule. The LCC for the time-dependent rule is larger than that for the condition-dependent rule, regardless of the risk control level. Accordingly, LCC can be reduced considerably, by inspecting the road condition regularly and adopting the condition-dependent rule. When the risk control level is high (the risk control level $\bar{U}$ is low), LCC reduction is large, and as the risk control level $\bar{U}$ 
Table 2 Optimal inspection/repair policy (extended model).

\begin{tabular}{c|c|c|c|c}
\hline Risk control & Optimal inspection & Regular inspection & \multicolumn{2}{|c}{ Critical soundness level } \\
\cline { 4 - 5 } level $\bar{U}$ & policy & interval [years] & $i^{*}$ & $i^{* *}$ \\
\hline 0.01 & extended & 1 & 6 & 5 \\
0.05 & extended & 1 & 7 & 6 \\
0.1 & extended & 4 & 7 & 5 \\
0.2 & basic & 3 & 7 & - \\
0.3 & basic & 4 & 7 & - \\
0.4 & basic & 6 & 7 & - \\
0.5 & basic & 9 & 7 & - \\
0.6 & basic & 10 & 7 & - \\
\hline
\end{tabular}

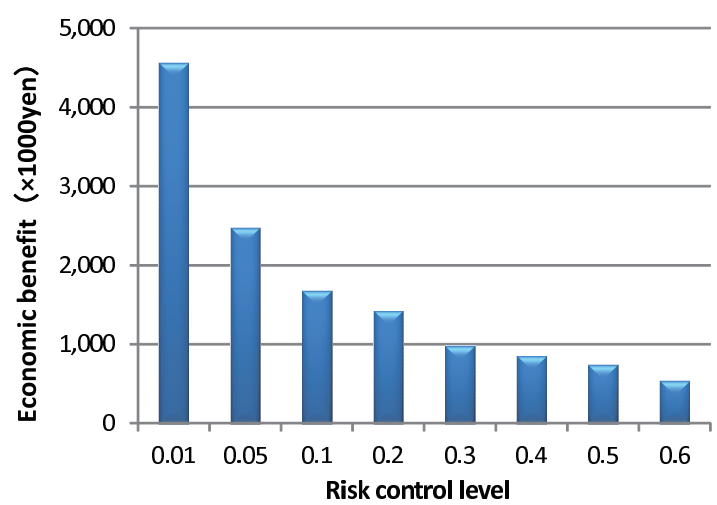

Fig.12 Economic benefit of road condition inspection.

increases, LCC reduction decreases. Thus, when we aim to keep the risk control level high, the economic value of the road condition inspection increases.

Fig.12 shows the results of the calculation of the economic benefits of the road condition inspection for each risk control level using Equation (45). On the other hand, Fig.13 shows the results of the calculation of the economic benefits of the additional inspection using Equation (49). When the risk control level is low, the economic benefits of the additional inspection become larger, and when the risk control level is over 0.2 , additional inspection is not carried out, and the economic benefits of additional inspection are not defined. Table $\mathbf{2}$ summarizes the optimal inspection/repair policy for each risk control level based on the extended model. These analysis results indicate that the additional inspection/repair policy is effective when risk control level is as rigorous as 0.1 or lower. When the risk control level is over 0.2 , the optimal solution of the basic model is equal to that of the extended model, and the additional inspection/repair policy is not implemented.

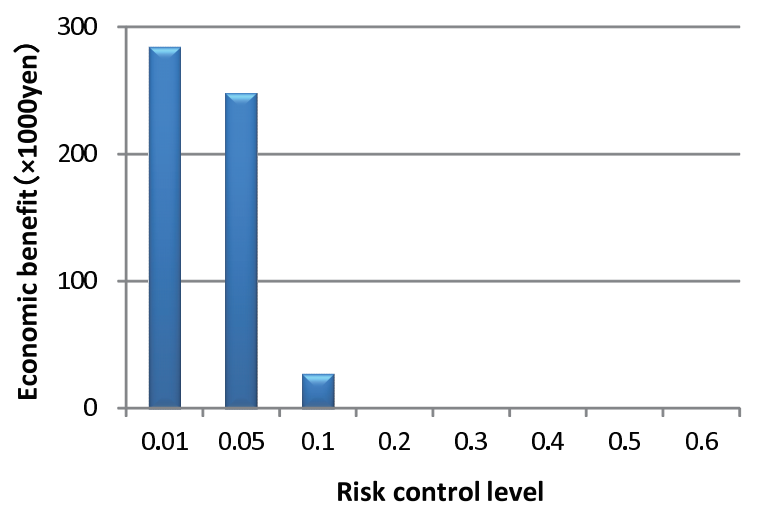

Fig.13 Economic benefit of additional inspection.

\section{CONCLUSIONS}

In this study, the authors proposed a methodology for specifying inspection interval and pavement repair policy that can minimize the lifecycle cost composed of the expenses for repair and inspection at the risk control level for pavement specified by road administrators. In detail, the authors expressed the deterioration process of pavement with a Markov deterioration prediction model and formulated the optimal inspection/repair model for determining inspection interval and repair policy that minimize the lifecycle cost at a specified risk control level. In addition, the authors proposed the extended model for determining a periodic inspection/repair policy and an additional inspection/repair policy for limited road spans. Furthermore, the economic benefit of the regular road condition inspection brought about by the shift of pavement repair policies from the time-dependent rule to the condition-dependent rule was mentioned, and a method for gauging the economic benefits of regular and additional inspections was proposed. Lastly, the effectiveness of the proposed methodology was analyzed empirically by discussing the case of the application to actual expressways. However, some subjects remain to be studied to 
improve the effectiveness of the proposed methodology. First, various methods for the additional inspection/repair policy can be considered, in addition to those discussed in this study. For example, it is assumed in this study that additional inspection is conducted every period, but it is acceptable to set the interval of additional inspection at over two periods. Such extension can be realized by modifying the formulation of the subMarkov decision model in the basic model. Second, it is assumed that the target road is constituted by spans with almost the same pavement properties and traffic characteristics. Accordingly, the authors formulated the optimal inspection/repair model using a single performance curve. If the target road is constituted by spans with various pavement structures and road characteristics, it is necessary to develop the optimal inspection/repair model considering the heterogeneity of road spans. Third, in the application case of this study, inspection/repair policies were discussed focusing on a single road. From now on, it will be necessary to study more than one road and develop a methodology for specifying practical road condition inspection. Fourth, the proposed optimal inspection/repair model can be applied to problems in the asset management of civil infrastructures other than pavements by modifying it a little. Especially, if repair methods are diverse, the number of repair policies becomes enormous, and so it is necessary to develop an efficient solving method.

\section{REFERENCES}

1) Aoki, K., Yamamoto, K. and Kobayashi, K.: Estimating Hazard Models for Deterioration Forecasting, Journal of Construction and Management, JSCE, No.791/VI-67, pp.111-124, 2005.

2) Tsuda, Y., Kaito, K., Aoki, K. and Kobayashi, K.: Estimating Markovian Transition Probabilities for Bridge Deterioration Forecasting, Journal of Structural Eng./Earthquake Eng., JSCE, Vol.23, No.2, pp.241s-256s, 2006.

3) Aoki, K., Yamamoto, K., Tsuda, Y. and Kobayashi, K.: A Deterioration Forecasting Model with Multi-Staged Weibull Hazard Functions, Journal of Construction and Management, JSCE, No.798/VI-68, pp.125-136, 2005.

4) Tsuda, Y., Kaito, K., Yamamoto, K. and Kobayashi, K.: Bayesian Estimation of Weibull Hazard Models for Deterioration Forecasting, Journal of Construction and Management, JSCE, Vol.62, No.3, pp.473-491, 2006.

5) Kaito, K. and Kobayashi, K.: Bayesian Estimation of Markov Deterioration Hazard Model, Journal of Structural Engineering 83 Earthquake Engineering, JSCE, Vol.63, No.2, pp.336-355, 2007.

6) Obama, K., Okada, K., Kaito, K. and Kobayashi,
K.: Disaggregated Hazard Rates Evaluation and Bench-Marking, Journal of Structural Engineering \& Earthquake Engineering, JSCE, Vol.64, No.4, pp.857-874, 2008.

7) Kobayashi, K., Kumada, K., Sato, M., Iwasaki, Y. and Aoki, K.: A Pavement Deterioraton Forcasting Model with Reference to Sample Dropping, Journal of Construction and Management, JSCE, Vol.63, No.1, pp.1-15, 2007.

8) Heyman, D. P. and Sobel, M. J. (eds.): Stochastic Models, Handbooks in Operations Research and Management Science, Vol.2, North-Holland, 1990.

9) Howard, R. A.: Dynamic Programming and Markovian Processes, The MIT Press, 1960.

10) Madanat, S.: Incorporating inspection decisions in pavement management, Transportation Research, Part B, Vol.27B, pp.425-438, 1993.

11) Madanat, S. and Ben-Akiva, M.: Optimal inspection and repair policies for infrastructure facilities, Transportation Science, Vol.28, pp.55-62, 1994.

12) Durango, P. and Madanat, S.: Optimal maintenance and repair policies for infrastructure facilities under uncertain deterioration rates: An adaptive control approach, Transportation Research, Part A, Vol.36, pp.763-778, 2002.

13) Kaito, K., Yasuda, K., Kobayashi, K. and Owada, K.: Optimal Maintenance Strategies of Bridge Components with an Average Cost Minimizing Principles, Journal of Structural Engineering E Earthquake Engineering, JSCE, No.801/I-73, pp.83-96, 2005.

14) Otazawa, T., Ishihara, K., Kobayashi, K. and Kondo, Y.: Optimal Repair Strategies with Reference to Economic Life Expectancy, Journal of Infrastructure Planning and Management, JSCE, No.772/IV-65, pp.169-184, 2004.

15) Aoki, K., Yamamoto, K. and Kobayashi, K.: Optimal Inspection and Replacement Policy of Tunnel Lighting Systems, Journal of Construction and Management, JSCE, No.805/VI-69, pp.105116, 2005.

16) Aoki, K., Yamamoto, K. and Kobayashi, K.: An Optimal Inspection/Rehabilitation Model of Multi-Components Systems with TimeDependent Detrioration Processes, Journal of Construction and Management, JSCE, Vol.62, No.2, pp.240-257, 2006.

17) Jido, M., Otazawa, T. and Kobayashi, K.: Synchronized Repair Policy for Bridge Management, in: Watanabe, E., Frangopol, D. M. and Utsunomiya, T. (eds.), Bridge Maintenance, Safety, Management and Cost, CD-ROM, Balkeme, 2005.

18) Jido, M. and Kobayashi, K.: The Optimal Repairing and Inspection Rules under Uncertainty, Journal of Infrastructure Planning and Management, JSCE, No.744/IV-61, pp.39-50, 2003.

19) Hayashi, H., Kaito, K., Kumada, K. and Kobayashi, K.: A Competitive Deterioration Hazard Model: Applying for Pavement Cracking Processes, Journal of Infrastructure Planning 
and Management, JSCE, Vol.65, No.2, pp.143$162,2009$.

20) Kumada, K., Eguchi, T., Aoki, K., Kaito, K. and Kobayashi, K.: A Pavement Deterioration Forecasting Model in Expressways based on Monitor
Data, Journal of Pavement Engineering, Vol.14, pp.229-237, 2009.

(Received October 17, 2013) 